\title{
Characterization and fine mapping of an early senescence mutant (es-t) in Oryza sativa L.
}

\author{
YANG YaoLong ${ }^{1 \dagger}$, RAO YuChun $^{1 \dagger}$, LIU HuiJuan $^{1,2}$, FANG YunXia $^{1}$, DONG GuoJun $^{1}$, \\ HUANG LiChao ${ }^{1}$, LENG YuJia ${ }^{1}$, GUO LongBiao ${ }^{1}$, ZHANG GuangHeng ${ }^{1}$, HU Jiang ${ }^{1}$, \\ GAO Zhen $\mathrm{Yu}^{1}$, QIAN Qian ${ }^{1 *} \&$ ZENG DaLi ${ }^{*}$ \\ ${ }^{1}$ State Key Laboratory of Rice Biology, China National Rice Research Institute, Hangzhou 310006, China; \\ ${ }^{2}$ College of Life and Environment Sciences, Hangzhou Normal University, Hangzhou 310036, China
}

Received March 9, 2011; accepted May 20, 2011

\begin{abstract}
An es-t (early senescence-temporary) mutant, produced by ethylene methylsulfonate treatment of strain Nipponbare, was identified in rice. The leaves of $e s-t$ appeared yellow at the seedling stage, and had decreased chlorophyll content. Rust spots were found during growth in $e s-t$, especially at the leaf margin and tip. The plants showed a typical early-senescence phenotype at the milky stage. The leaf surface of $e s-t$ appeared smoother than wild-type leaves under a scanning electron microscope, because the leaves lack siliceous protuberances around the stoma. Chloroplasts grow abnormally and are filled with many starch grains in $e s-t$. Paraffin section analysis showed that the development of the sclerenchyma cells and vascular bundles were also abnormal in $e s-t$. Genetic analysis indicated that $e s-t$ was controlled by a recessive gene, which was finely mapped to a $42-\mathrm{kb}$ interval on chromosome 5. These results will facilitate the positional cloning and functional studies of the gene.
\end{abstract}

Oryza sativa, morphological and physiological analysis, genetic mechanism, map-based cloning

Citation: $\quad$ Yang Y L, Rao Y C, Liu H J, et al. Characterization and fine mapping of an early senescence mutant (es-t) in Oryza sativa L. Chinese Sci Bull, 2011, 56: 2437-2443, doi: 10.1007/s11434-011-4587-8

Leaves are the primary organs of plants for performing photosynthesis and respiration, and their development is closely related to agricultural yield [1,2]. Leaf senescence in rice is one of the limiting factors for developing high yield potential [3]. When plants are under stress, they can exhibit a type of leaf senescence called programmed cell death, which aids germplasm survival [4]. The early senescence phenomenon in rice leads to incomplete grain filling and reductions in the parameters of setting percentage and 1000 -grain weight. In some hybrid rice varieties, early senescence results in the shortening of the leaf functional period and reduced photosynthate accumulation, which ultimately has a severe effect on rice yield. Research has shown that yield can be increased if senescence can be postponed $[5,6]$. The discovery of relevant mutants is im-

\footnotetext{
$\dagger$ These authors contributed equally to this work.

* Corresponding authors (email: qianqian188@hotmail.com; dalizeng@126.com)
}

portant for elucidating the molecular mechanism of senescence, regulating the senescence process, and cultivating antisenescence varieties.

To date, 30 senescence-associated genes have been cloned from different plant groups. Genes including WRKY/NAC, transcription factors, hydrolyzed proteases, acyltransferases, and auxin response factors have been cloned and functionally analyzed in Arabidopsis thaliana. Such discoveries are crucial to revealing the mechanism of leaf senescence and for understanding the complex regulatory network [7]. Eight senescence-associated genes have been cloned in rice, among which 6 (Os12 [8], Osh69 [9], $\operatorname{sgr}(t)$ [10], SGR [11], sgr [12], and nycl [13]), show upregulated expression and 2 (OsDos [14] and ygll [15]) show downregulated expression. Os12 and Osh69, encoding GABA aminotransferase and $\alpha$-galactosidase, respectively, were screened from senescent rice leaves through suppression 
subtractive hybridization. They are specifically expressed in senescent leaves, but hardly at all in the stem or root. GABA aminotransferase is an important enzyme in the mitochondrial GABA branch, and is closely related to proline metabolism. It also has an important role in carbon and nitrogen metabolism, and can prevent the accumulation of reactive oxygen species, emphasizing its importance in the plants defense mechanism. OsDos encodes a CCCH-type zinc finger protein and shows decreased expression during the panicle initiation and leaf senescence stages. Plants exhibit normal vegetative growth after OsDos interference. Leaves quickly turn yellow at a later ear period and all leaves are senescent at the grain-filling stage, except the flag leaves. Another underactive gene is $y g l l$, which was obtained through map-based cloning. The mutation obstructs the last step of esterification in the process of chloroplasts synthesis. Mutants' leaves turn yellow at the seedling stage and cannot grow normally [15]. Rice senescence is always accompanied by changes in leaf color. Sometimes, rust spots appear. Many genes related to spot and leaf color have been cloned, among which a spot mutant, $s p l 28$, that has the characteristic of early senescence, has been cloned [16]. The mutant has spotted leaves and the leaves senesce rapidly after flowering. The mutant withers at the later flowering stage and dies during the maturation stage.

In this study, the novel early senescence temporary $(e s-t)$ mutant was used as research material to study various morphological and physiological changes in rice plants resulting from the es- $t$ mutation. Map-based cloning located the $E S-t$ gene to a $42.1-\mathrm{kb}$ DNA region. The study provides the basis for further cloning of this gene, for elucidating the mechanism of early senescence, and for regulating the process of leaf senescence for improved agricultural production.

\section{Materials and methods}

\subsection{Plant materials}

The mutant $e s-t$ was obtained from Nipponbare rice and subjected to mutagenesis with ethylene methylsulfonate (Sigma Corporation, USA). Crosses were made between $e s-t$ and conventional varieties $\mathrm{TN}_{1}, \mathrm{ZF} 802$, and NJ06. The seeds from the $F_{1}$ and $F_{2}$ generations were used for further research. The $\mathrm{F}_{2}$ individuals with the mutant phenotype were used for gene mapping.

\subsection{Detection of chlorophyll content}

Fresh leaves from Nipponbare and $e s-t$ during their active tillering stage were sampled and the leaf veins removed. Leaves $(0.3 \mathrm{~g})$ were cut into $1 \mathrm{~cm}$ segments, placed into a small bottle and soaked in $80 \%$ acetone (Huadong Medicine Corporation, Hangzhou, China) for $48 \mathrm{~h}$ at $26^{\circ} \mathrm{C}$ in dark conditions. The optical density of the resultant solution was measured spectrophotometrically (Du800, BECKMAN COULTER, German) at wavelengths of 470, 645 and 663 $\mathrm{nm}$. Each sample was measured 3 times. The formulas [17] for calculating the total chlorophyll, chlorophyll $a(\mathrm{Chl} a)$, chlorophyll $b(\mathrm{Chl} b)$, and carotenoid contents were:

Chl $a=\left(12.7 \times A_{663}-2.69 \times A_{645}\right) \times V / W$;

Chl $b=\left(22.9 \times A_{645}-4.68 \times A_{663}\right) \times V / W$;

Car $=\left(1000 \times A_{470} \times V / W-3.27 \times \mathrm{Chl} a-104 \times \mathrm{Chl} b\right) / 198$.

\subsection{Scanning and transmission electron microscopy}

Middle-upper Nipponbare and es-t leaves were selected during their trefoil stage. The leaves were cut into $0.5-1 \mathrm{~cm}$ segments and immediately placed into a precooled glutaraldehyde (Huadong Medicine Corporation) stationary liquid (2.5\%) for fixation. The segments were washed 3 times in the phosphate buffer solution (composed of $\mathrm{Na}_{2} \mathrm{HPO}_{4}$ and $\mathrm{NaH}_{2} \mathrm{PO}_{4}, 0.1 \mathrm{~mol} / \mathrm{L} \mathrm{PO}_{4}{ }^{-3}, \mathrm{pH}$ 7.0, Huadong Medicine Corporation) and treated overnight in $1 \%$ osmic acid solution. After being washed in the phosphate buffer solution, the samples were dehydrated using a gradient of ethanol solutions from 50\% to 95\% (Huadong Medicine Corporation). The treated sample were used for Scanning and transmission electron microscopy. For scanning electron microscopy, transition was conducted using $100 \%$ isoamyl acetate (Huadong Medicine Corporation) for $1-2 \mathrm{~h}$ before performing $\mathrm{CO}_{2} \mathrm{HCP}-2$ critical point drying. Carbon coating was performed using an $\mathrm{H} 5 \mathrm{~GB}$ vacuum spray plating instrument (Hitachi Corporation, Japan). Observation was performed on a Hitachi TM-1000 scanning electron microscope (Hitachi Corporation).

For transmission electron microscopy, the acrylic acid resin (Huadong Medicine Corporation) was applied to permeate and embed the samples. Sections were cut using an ultramicrotome (Reichert Corporation, German). Sections were stained using uranium acetate/lead citrate double staining (Huadong Medicine Corporation) and then observed on a Hitachi H-7650 transmission electron microscope (Hitachi Corporation).

\subsection{Cytological observation of leaves}

One week after heading, the middle-upper part of Nipponbare and es- $t$ leaves were sampled and cut into 1-2 mm segments. The segments were placed into FAA solution (formalin-aceto-alcohol solution) for $24 \mathrm{~h}$ and stored at $4{ }^{\circ} \mathrm{C}$ for later use. The segments were dehydrated, made transparent, wax-filled, and embedded. Conventional paraffin sectioning was used and staining was conducted using safranine and fast green (Huadong Medicine Corporation). Samples were mounted on a slide and covered with a coverslip before observation on an optical microscope (HM340E, German). 


\subsection{Genetic analysis of mutant $e s-t$}

According to previous studies, es- $t, d w l 1$ [18], spl-7 [19], spl-11 [20], and spl-28 [16] have similar phenotypes; thus, crosses between es- $t$ and these other mutants ( $d w l l, s p l-7$, spl-11, spl-28) were made. The phenotypes of the $\mathrm{F}_{1}$ and $\mathrm{F}_{2}$ generations of different crosses were recorded. The separation ratios were calculated to determine whether they were allelic. Meanwhile, reciprocal crosses between es- $t$ and common varieties $\left(\mathrm{TN}_{1}, \mathrm{ZF} 802\right.$ and NJ06) were made to study the genetic mechanism of es-t. The software SAS 8.0 was used to compute the chi-square value.

\subsection{Gene mapping of $E S$ - $t$}

The early senescence pool and the normal pool were constructed by the BSA (bulked segregant analysis) method. Polymorphism analysis of the parents of the segregation population was accomplished using simple sequence repeat (SSR) markers. The $\mathrm{F}_{2}$ generation was used to screen polymorphic markers to analyze linkage groups of the ES- $t$ gene and the linked markers were carried through to linkage analysis of the mutant individuals. The simple sequence repeat (SSR) markers were uniformly distributed over the 12 rice chromosomes. The PCR reaction method was according to Panaud et al. [21] and used an EDC-810 PCR machine (Dongsheng Biotech Corporation). The PCR products were subjected to $4 \%$ agarose gel electrophoresis and the result was observed and recorded using a gel imaging system (ALphalmager EP, Alpha Innotech Corporation, USA) after staining with ethidium bromide. Primer Premier 5 software was used to design primers for sequence-tagged site markers and cleaved amplified polymorphic sequences markers, according to the sequence differences between japonica (http://rgp.dna.affrc.go.jp) and indica var. 93-11 (http://www.rise.genomics. org.cn) on chromosome 5. The primers were used for fine mapping of the ES- $t$ gene.

\section{Results}

\subsection{Morphological characteristics of $e s-t$}

The distinguishing feature of es- $t$ is the yellowing of the leaves from the seedling stage. The leaves turn yellow and rust spots appear. Notably, the leaf margins and tips are more seriously affected than the other parts (Figure 1(a)) and show serious early senescence symptoms at the heading stage. Rust spots can also be seen on mature grains, half of which appear to have open glumes and are slightly smaller than normal (Figure 1(b)). The root system grows slower than that of the wild-type after seed germination. The main taproot is shorter than that of wild-type and has fewer lateral roots (Figure 1(c)). In particular, on the 5th day after seed germination, the length of main root in the mutant was 2.80 $\mathrm{cm}$, while the length of main root in the wild-type was 5.87 $\mathrm{cm}$ (Figure 1(e)). The ES- $t$ mutant may affect rice yield because of the sharp decrease in the number of primary branches (Table 1). However, the mutation has little effect on plant height (Figure 1(d)). In addition, the mutant also has an effect on the tillering capacity of rice (Table 1). During active tillering, wild-type individuals have 12 tillers, while mutant individuals have about 7 tillers.

Table 1 Comparison of major agronomic traits between es- $t$ and wildtype rice

\begin{tabular}{crrcc}
\hline $\begin{array}{c}\text { Agronomic } \\
\text { traits }\end{array}$ & Tiller no. & $\begin{array}{c}\text { Number of } \\
\text { primary } \\
\text { branches }\end{array}$ & $\begin{array}{c}\text { Number of } \\
\text { grains per ear }\end{array}$ & $\begin{array}{l}1000 \text {-grain } \\
\text { weight }(\mathrm{g})\end{array}$ \\
\hline Wild type & $12.3 \pm 3.09$ & $8.4 \pm 2.32$ & $94.2 \pm 25.53$ & $22.8 \pm 0.207$ \\
$e s-t$ & $7.6 \pm 2.50$ & $6.3 \pm 1.89$ & $53.1 \pm 13.44$ & $19.7 \pm 0.274$ \\
\hline
\end{tabular}
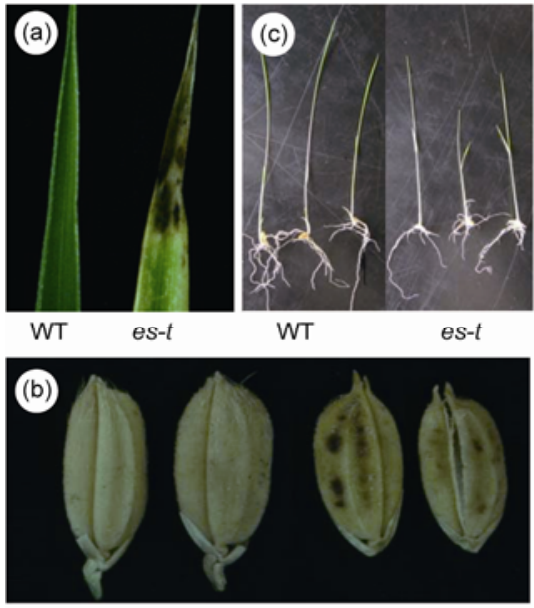

WT

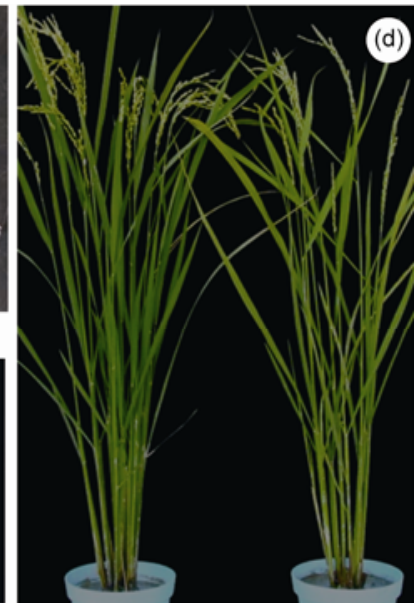

WT

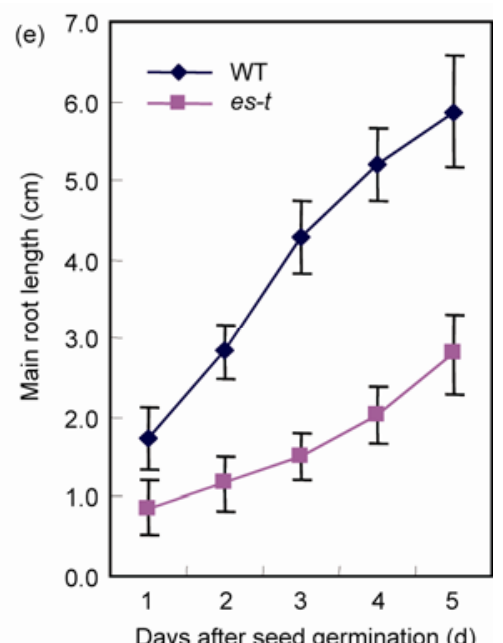

Days after seed germination (d)

Figure 1 The morphological differences between wild-type and $e s-t$ plants. (a) Top of flag leaves during maturation stage; (b) rice grain; (c) sand-cultured rice seedlings; (d) rice plant at the maturation stage; and (e) dynamic changes in the length of main root in young seedlings. 


\subsection{Genetic analysis of mutant es-t}

The mutant stably exhibited the mutant phenotype after several generations of continuous selfing. Crosses between $e s-t$ and other mutants with similar phenotypes ( $d w l 1, s p l-7$, spl-11, and spl-28) suggested that es- $t$ is not allelic with any of those genes. The $4 \mathrm{~F}_{1}$ population exhibited wild-type phenotype, and there were no es- $t$ individuals in all $\mathrm{F}_{1}$ population. In all $\mathrm{F}_{2}$ generation, the chi-square values were smaller than $3.84\left(\chi_{0.05,1}^{2}=3.84\right)$, the ratio of normal phenotype to early senescence in every combination was close to 9:7 (Table 2). These results indicated that es- $t$ was nonallelic to other 4 genes.

The phenotype investigation of 6 population were shown in Table 3. All $F_{1}$ populations derived from the reciprocal crosses between $e s-t$ and other common varieties $\left(\mathrm{TN}_{1}\right.$, ZF802, and NJ06) showed normal phenotypes. These results indicated that $e s-t$ is controlled by nuclear gene. Further analysis of their phenotypes in the $F_{2}$ generation showed that the ratio of wild-type phenotype to early senescence in every combination was close to $3: 1$. These results also indicated that the characteristic is qualitative and controlled by a single recessive nuclear gene.

\subsection{Chlorophyll content}

Leave senescence always leads to decreases in chlorophyll content. We detected the total chlorophyll, chlorophyll $a$, and chlorophyll $b$ contents in both es- $t$ and in Nipponbare (wild-type) leaves at their active tillering stage. The results indicated that the phenomenon of yellowing leaves is caused by decreasing amounts of chlorophyll (Figure 2). Leaves of es- $t$ contained less total chlorophyll, chlorophyll $a$, chlorophyll $b$, and carotenoid than those of the wild-type. Among which, the decrease in chlorophyll $a$ content was the lowest: about $60 \%$ of the wild type level. However, the relative ratio of chlorophyll $a$ to chlorophyll $b$ changed very little (approximately 3:1).

\subsection{Scanning electron microscope analysis}

Scanning electron microscopy revealed that es- $t$ has a smoother leaf surface than that of the wild-type. The leaf hairs on the leaf surface are sparse in es-t. The wild-type leaves have many spinous processes on their leaf surfaces,

Table 2 Genetic analysis of $F_{2}$ populations derived from crosses between $e s-t$ and other similar mutants

\begin{tabular}{ccccccc}
\hline \multirow{2}{*}{ Combinations } & \multicolumn{3}{c}{$\mathrm{F}_{1}$} & & \multicolumn{3}{c}{$\mathrm{F}_{2}$} \\
\cline { 2 - 3 } \cline { 5 - 6 } & wild type & $e s-t$ & & wild type & es- $t$ & $\chi^{2}(9: 7)$ \\
\hline$e s-t / d w l 1$ & 17 & 0 & 360 & 258 & 1.0069 \\
$e s-t / s p l-7$ & 16 & 0 & & 420 & 325 & 0.0048 \\
$e s-t / s p l-11$ & 15 & 0 & & 378 & 273 & 0.871 \\
$e s-t / s p l-28$ & 13 & 0 & 323 & 244 & 0.1183 \\
\hline
\end{tabular}

(a)

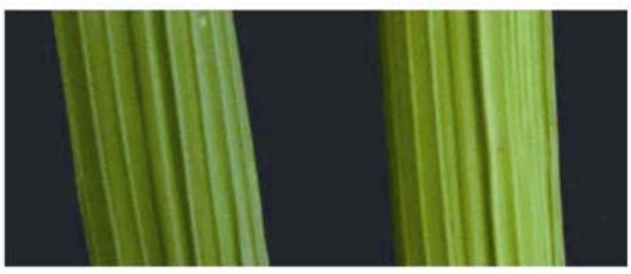

WT

$e s-t$

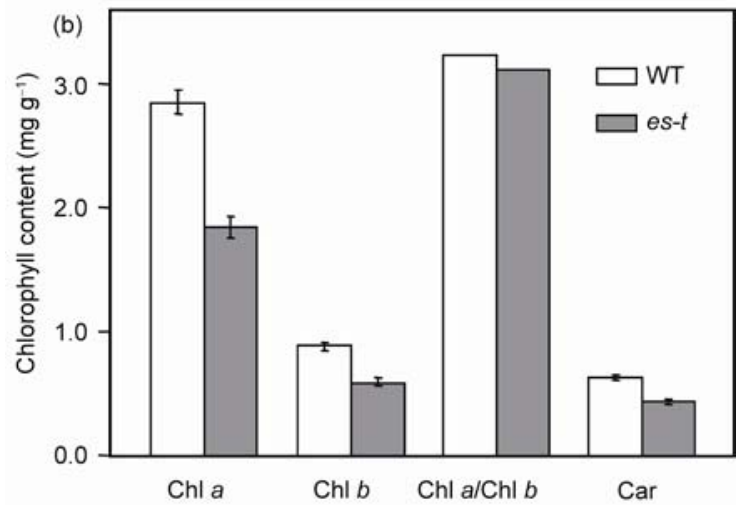

Figure 2 Comparisons of wild-type and $e s-t$ plants for flag leaf color (a) and chlorophyll content in the flag leaf (b).

Table 3 Genetic analysis of $F_{2}$ populations derived from crosses between $e s-t$ and other common varieties

\begin{tabular}{|c|c|c|c|c|c|}
\hline \multirow{2}{*}{ Combinations } & \multicolumn{2}{|c|}{$\mathrm{F}_{1}$ population } & \multicolumn{2}{|c|}{$\mathrm{F}_{2}$ population } & \multirow{2}{*}{$\chi^{2}(3: 1)$} \\
\hline & Wild type & $e s-t$ & Wild type & $e s-t$ & \\
\hline$e s-t / \mathrm{TN}_{1}$ & 28 & 0 & 526 & 167 & 0.3006 \\
\hline $\mathrm{TN}_{1} / e s-t$ & 18 & 0 & 451 & 142 & 0.3513 \\
\hline es-t/ZF802 & 9 & 0 & 245 & 70 & 1.2963 \\
\hline ZF802/es- $t$ & 12 & 0 & 163 & 47 & 0.7683 \\
\hline$e s-t / \mathrm{NJ} 06$ & 8 & 0 & 385 & 116 & 0.9108 \\
\hline NJ06/es- $t$ & 16 & 0 & 365 & 107 & 1.3672 \\
\hline
\end{tabular}

whereas the number of spinous processes was significantly reduced in $e s$ - $t$ (Figure 3(a) and (b)). In addition, the number of silicified processes on the leaf surface of $e s-t$ was significantly smaller than in wild-type. There was no obvious change in the number of stomas. No silicified processes were found around the stomas in $e s-t$, which differed markedly from the wild-type (Figure 3(c) and (d)). The presence of silicified processes around stomas may be related to the silicide deposition caused by transpiration of stomas and has a certain influence on the growth and development of rice.

\subsection{Transmission electron microscope analysis}

The chlorophyll content in the early senescence leaves of $e s-t$ is less than that of the wild type. Transmission electron microscopy was used to compare the structure of the mesophyll cells between Nipponbare and es- $t$. The results indicatedy that es- $t$ has fewer chloroplasts than the wild-type, and its chloroplasts are smaller than wild-type. In 
addition, the chloroplasts are irregular and slender in $e s-t$ (Figure 4(a) and (b)). The chloroplasts in $e s$ - $t$ contain many large granular starch grains, which are not found in the wild-type (Figure 4(c) and (d)). This may explain the reduced chlorophyll content and yellowed leaves in $e s-t$.

\subsection{Abnormal development of vascular bundles in $e s-t$}

Both normal leaves and early senescence leaves were subjected to paraffin sectioning to further understand the formation mechanism of early senescence leaves. Compared with wild-type leaves, es- $t$ has fewer developed vascular bundles in the main veins (Figure 5(a) and (b)). This may account for the reduced transport capacity of water and minerals that leads to early senescence of the leaves. Fewer vesicles, which are located between the vascular bundles, and sclerenchymatous cells, were found in the leaf margin of $e s$ - $t$, compared with those of the wild-type. This accounts for the difference between $e s-t$ leaves and normal leaves at the leaf margin.
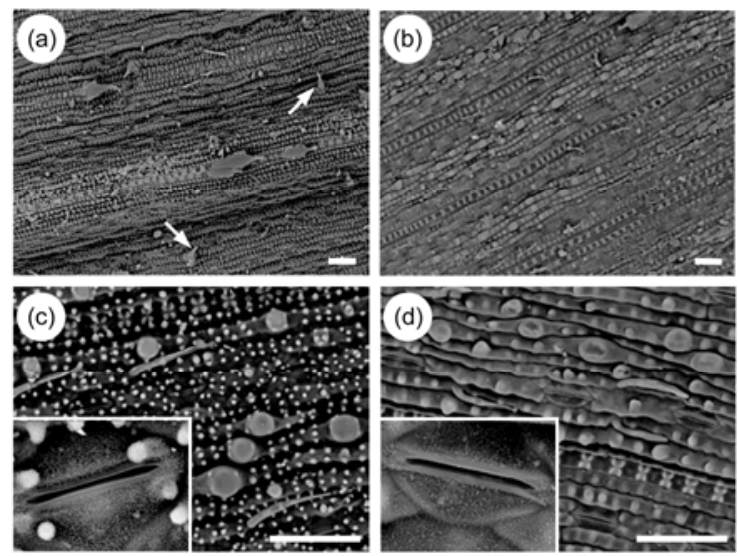

Figure 3 The difference between the leaf surfaces of wild-type and $e s-t$ plants as determined by scanning electron microscopy. (a) and (b) The smoother leaf surface exit in $e s-t$; (c) and (d) the absence of silicified processes around the stomas in $e s-t$. Bar $=50 \mu \mathrm{m}$.
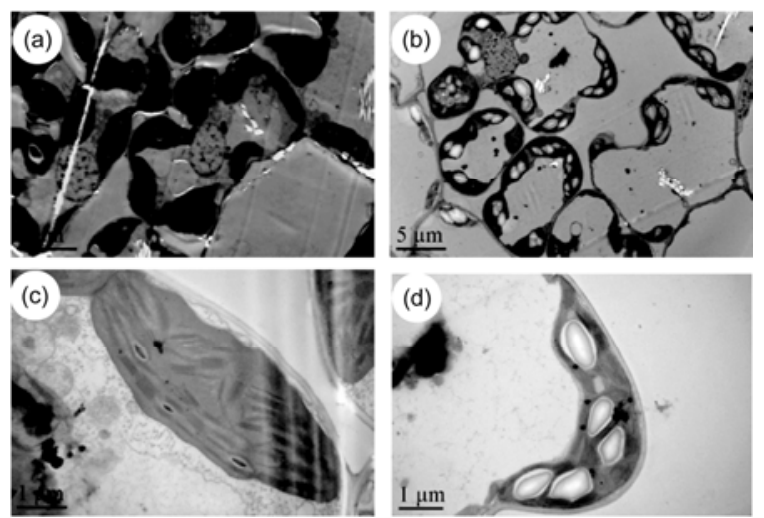

Figure 4 The differences between the chloroplasts of wild-type ((a) and (c)) and es-t ((b) and (d)) plants as determined by transmission electron microscopy.
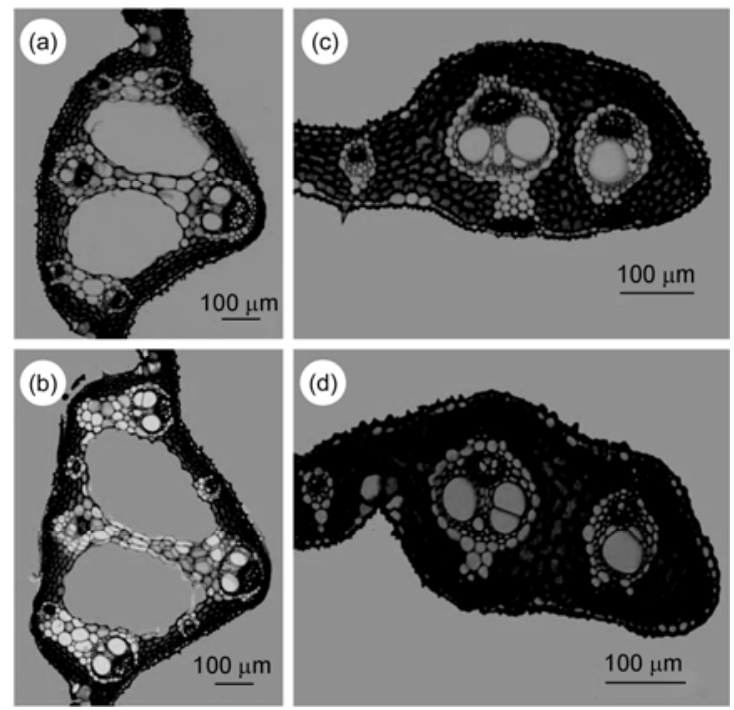

Figure 5 Transverse-section comparison of wild-type and es- $t$ leaves using paraffin sectioning for the main veins and leaf margins of es- $t$ ((a) and (c), respectively) and wild-type ((b) and (d), respectively).

\subsection{Fine mapping of $E S-t$}

Ninety-six pairs of SSR markers, uniformly distributed over the 12 rice chromosomes with intervals ranging from 10-20 cM, were selected to detect amplification diversity between $\mathrm{F}_{1}$ genomic DNA and mutant pool DNA for primary mapping of the target gene. Marker RM3809, locatedon chromosome 5, exhibited significant amplification diversity between $\mathrm{F}_{1}$ genomic DNA and mutant pool DNA. Twenty individuals that were used to construct the mutant pool were used to test whether RM3809 was linked with the target gene phenotype and the result was positive. New markers were developed close to RM3809 for PCR amplification and detection. The results showed that the molecular marker RM334 is also linked with the target gene and its recombinant individuals are different from those of RM3809. This indicated that the ES- $t$ gene is located between markers RM3809 and RM334. From among 265 mutant individuals separated using RM3809 and RM334, 19 and 23 recombinant individuals were detected, giving calculated genetic distances between the ES- $t$ gene and RM3809 and RM334 of 3.5 and $4.3 \mathrm{cM}$, respectively.

For fine mapping, SSR markers were developed between RM3809 and SSR334 (Table 4). A total of 2551 $\mathrm{F}_{2}$ individuals with the mutant phenotype were selected for further mapping. The gene was located in a 42.1-kb DNA region between markers P3 and P6 (Figure 6(b) and (c)). This region contains 6 ORFs (open reading frames): the 50S ribosomal protein L21 in the chloroplastic precursor (LOC_Os05g48410), a component of a membrane-bound complex (LOC_Os05g48360), two ubiquitin conjugating enzyme genes (LOC_Os05g48380, LOC_Os05g48390), and two proteins of unknown function (LOC_Os05g48370, LOC_Os05g48400). 
Table 4 Markers for $E S$ - $t$ gene mapping

\begin{tabular}{cll}
\hline Primer & \multicolumn{1}{c}{ Forward primer $\left(5^{\prime} \rightarrow 3^{\prime}\right)$} & \multicolumn{1}{c}{ Reverse primer $\left(5^{\prime} \rightarrow 3^{\prime}\right)$} \\
\hline RM3809 & AAATATCTATCGGCCTCTCCAAGC & GGAGGAATCGAACCAGAGAAGC \\
RM334 & GCCGGTAGATGCAGTATTGC & GTGCACAGTCACGCAGAAAG \\
P1 & TTTCAAGGACTATCTAAACCG & CAAGAGGTTTGTGCTGTTTA \\
P2 & GAGTCGGTAATCAGGTTTT & AATCGTGAACATGAAGCA \\
P3 & CAATTAAGTCATTTGCCTAG & TAGTAACGTGTTCTTCGA \\
P4 & TTTTCTTGCTCCTCTTGG & AAATGCTACAGATGACGGA \\
P5 & ATCCGAGTGAAGGGAAATG & GCCTCAAAGCACCAAACA \\
P6 & CCAAAGTCTTGTAGGGG & CATATCAAAGTTAGTTAGCG \\
P7 & TGTAGTGTATTCCAAGCCTCT & CTGTTATTACCGCCTGACC \\
P8 & TCCGCATCGTATCTCCACAT & AGTCCCGGAGCGACCCATC \\
\hline
\end{tabular}

\section{Discussion}

In this study, we identified a novel early senescence mutant es-t. es-t plants show slightly lower plant height and its leaves show rust spots and senescence. Tissue sections showed abnormally developed vascular bundles in es-t. The structural abnormalities underlying the phenotype of the mutant may affect the photosynthetic efficiency and the absorption and utilization of organic matter in leaves. This in turn will lead to the suppression of rice growth and a decrease in biomass. A large number of starch grains were found in the chloroplasts of the es- $t$ mutant. Sucrose produced by photosynthesis is transported to different parts of the plant in the form of sucrose-Pi. Sucrose-Pi is stored in cytoplasmic amyloplasts in the form of starch grains to maintain the normal growth and development of the plant. On the other hand, other growth areas that lack the energy needed for normal growth may exhibit the early senescence phenotype.

The stoma is a specialized structure located on the leaf surface. They normally have silicified processes on guard cells [22]. The silicified epidermis generally acts as a barrier and has a function of mechanical protection. The silicification characteristic of the epidermis is related to stomatal opening and closing, which links photosynthesis and transpiration in rice [23]. Scanning electron microscope images

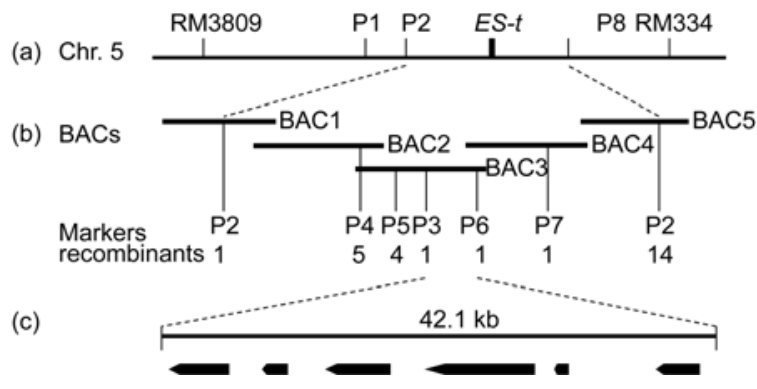

Figure 6 Fine mapping of $E S$ - $t$. (a) $E S$ - $t$ was located in the region between marker P2 and P8; (b) ES- $t$ was mapped to the region between marker P3 and P6; (c) ES- $t$ was mapped to a 42.1-kb region with 6 candidate genes in it. showed a significant decrease in the number of silicified particles on the surface of the mutant leaves. A previous report suggested that a reduction in silicified particles results in short plant height, significant decrease in resistance, affects root growth, and leads to a decline in the seed setting rate [24].

Some studies have indicated that mutation of the $50 \mathrm{~S}$ ribosomal protein in chloroplastic precursors may influence the normal programmed senescence of leaves. For example, the overexpression of SGR influences the binding of thylakoid membrane protein in chloroplasts, which leads to early senescence or the stay-green phenomenon in plants $[10,11]$. There is a gene encoding the $50 \mathrm{~S}$ ribosomal protein L21 in the chloroplastic precursor (LOC_Os05g48410) in the mapped region containing the ES- $t$ gene. According to recent study, a functional deficiency in ubiquitin conjugating enzyme gene LTN1 (LOC_Os05g48390) leads to an excessive accumulation of phosphorus, which results in yellow leaves, a symptom of phosphorus poisoning [25-27]. Ubiquitination is a type of epigenetic modification. Protein ubiquitination results in the specific degradation of the protein itself. Protein degradation is the main reaction during leaf senescence [28]. Two ubiquitin conjugating enzyme genes (LOC_Os05g48380, LOC_Os05g48390) are found in the $E S$-T region. Except for the withered leaf-tip, which is similar to $\ln 1$, the es- $t$ mutant exhibits leaf yellowing at the young seedling stage, which is different from $l$ tn 1 . Whether the mapped $E S-T$ gene is a homologous gene of known function or is a new gene encoding a protein of unknown function should be determined by sequence analysis and functional complementation assays. The fine mapping of $E S-T$ presented here lays the foundation for these future studies.

This work was supported by the National Special Program for Research and Transgenic Plants (2011ZX08009-003), the National Key Basic Research Program of China (2007CB10920203), and the National Natural Science Foundation of China (30971760).

1 Yan S, Yan C J, Gu M H. Molecular mechanism of leaf development 
(in Chinese). Hereditas, 2008, 30: 1127-1135

2 Shen N W, Qian Q, Zhang G H. Research progress on rice rolled leaf and its application in breeding program (in Chinese). Mol Plant Breed, 2009, 7: 852-860

3 Cao X Z, Zhu Q S. Pilot experiment to improve the setting percentage of hybrid rice (in Chinese). Jiangsu Agric Sci, 1981, 18: 5-6

4 Chae H S, Lee W S. Ethylene and enzyme-mediated superoxide production and cell death in carrot cells grown under carbon starvation. Plant Cell Rep, 2001, 20: 256-261

5 Wang X J, Xu Q G, Yang Z J. Advances of research on rice leaf senescence physiology (in Chinese). Chinese Agric Sci Bull, 2005, 21: 187-191

6 Liang J S, Cao X Z. Studies of the relationship between several physiological characteristics of leaf and bleeding rate of roots in hybrid rice (O. sativa L.) (in Chinese). Jiangsu Agric Sci, 1993, 14: 25-30

7 Balazadeh S, Riaño-Pachón M D, Mueller-Roeber B. Transcription factors regulating leaf senescence in Arabidopsis thaliana. Plant Biol, 2008, 10: 63-75

8 Ansari M I, Lee R H, Chen S C G. A novel senescence-associated gene encoding GABA: Pyruvate transaminase is up-regulated during rice leaf senescence. Physiol Plant, 2005, 123: 1-8

9 Lee R H, Wang C H, Huang L T, et al. Leaf senescence in rice plants: Cloning and characterization of senescence up-regulated genes. J Exp Bot, 2001, 52: 1117-1121

10 Cha K W, Lee Y J, Koh H J, et al. Isolation, characterization, and mapping of stay green mutant in rice. Theor Appl Genet, 2002, 104: 526-532

11 Jiang $\mathrm{H}$, Li M, Liang N, et al. Molecular cloning and function analysis of the stay green gene in rice. Plant J, 2007, 52: 197-209

12 Park S Y, Yu J W, Park J S, et al. The senescence-induced staygreen protein regulates chlorophyll degradation. Plant Cell, 2007, 19: $1649-1664$

13 Kusaba M, Ito H, Morita R, et a1. Rice NON-YELLOW COLORING1 is involved in light-harvesting complex II and grana degradation during leaf senescence. Plant Cell, 2007, 19: 1362-1375

14 Kong Z, Li M, Yang W, et al. A novel nuclear localized CCCH-type zinc finger protein, OsDOS, is involved in delaying leaf senescence in rice. Plant Physiol, 2006, 141: 1376-1388

15 Wu Z, Zhang X, He B, et a1. A chlorophyll-deficient rice mutant with impaired chlorophyllide esterification in chlorophyll biosynthe- sis. Plant Physiol, 2007, 145: 29-40

16 Qiao Y L, Jiang W Z, Lee J H, et al. SPL28 encodes a clathrin-associated adaptor protein complex 1 , medium subunit $u 1$ (AP1M1) and is responsible for spotted leaf and early senescence in rice (Oryza sativa). New Phytol, 2009, 185: 258-274

17 Amon D I. Copper enzymes in isolated chloroplasts: Polyphenoloxidase in Beta vulgaris. Plant Physiol, 1949, 24: 1-15

18 Jiang L, Guo L, Jiang H, et al. Genetic analysis and fine-mapping of a dwarfing with withered leaf-tip mutant in rice. J Genet Genomics, 2008, 35: 715-721

19 Kojo K, Yaeno T, Kusumi K, et al. Regulatory mechanisms of ROI generation are affected by rice $s p l$ mutations. Plant Cell Physiol, 2006, 47: 1035-1044

20 Zeng L R, Qu S, Bordeos A, et al. Spotted leaf11, a negative regulator of plant cell death and defense, encodes a U-Box/Armadillo repeat protein endowed with E3 ubiquitin ligase activity. Plant Cell, 2004, 16: $2795-2808$

21 Panaud O, Chen X, McCouch S R. Frequency of microsatellite sequences in rice (Oryza sativa L.). Genome, 38: 1170-1176

22 Zhang L P, Chen L B. Electron microscope observation of surface structure in rice leaves (in Chinese). J Chinese Electron Microscopy Soc, 1990, 9: 43

23 Shen H S, Chen J C, Huang J H, et al. Microstructure and distribution of silica bodies in rice epidermis (in Chinese). J Fujian Agric Univ (Nat Sci Ed), 2005, 34: 137-140

24 Yang B Y, Chen X F, Liu X D, et al. Observation of silicon cells on the leave surface in different varieties of rices (in Chinese). J Chinese Electron Microscopy Soc, 2006, 25: 146-149

25 Aung K, Lin S I, Wu C C, et al. pho2, a phosphate overaccumulator, is caused by a nonsense mutation in a microRNA399 target gene. Plant Physiol, 2006, 141: 1000-1011

26 Bari R, Pant B D, Stitt M, et al. PHO2, microRNA399, and PHR1 define a phosphate-signaling pathway in plants. Plant Physiol, 2006, 141: 988-999

$27 \mathrm{Hu} \mathrm{B}$, Zhu C G, Li F, et al. LEAF TIP NECROSIS 1 plays a pivotal role in regulation of multiple phosphate starvation responses in rice. Plant Physiol, 2011, doi: 10.1104/pp.110.170209

28 Zhang L X, Zong R J. Changes of respiration, and the contents of ethylene, IAA and peroxidase and their interrelationships during senescence of four leafy vegetables (in Chinese). J Plant Physiol Mol Biol, 1988, 14: 81-87

Open Access This article is distributed under the terms of the Creative Commons Attribution License which permits any use, distribution, and reproduction in any medium, provided the original author(s) and source are credited. 